\title{
Predictors of Taxonomic Inflation and its Role in Primate Conservation
}

Maria J.A. Creighton ${ }^{\mathrm{a}}$, Alice Q. Luo ${ }^{\mathrm{a}}$, Simon M. Reader ${ }^{\mathrm{a}} \&$ Arne Ø. Mooers ${ }^{\mathrm{b}}$

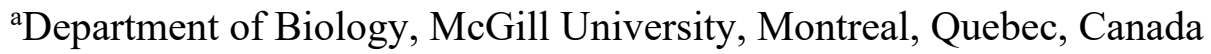

bepartment of Biological Sciences, Simon Fraser University, Burnaby, British Columbia, Canada

*Author for correspondence: maria.creighton@mail.mcgill.ca; ph: +1-514 398 7020; McGill University, Stewart Biology Building 1205 ave Docteur Penfield, Montreal, QC Canada, H3A 1B1 ORCID: https://orcid.org/0000-0003-1337-1946

WORD COUNT: 4941

DECLARATIONS OF INTEREST: None 


\section{ABSTRACT:}

2 Species are the main unit used to measure biodiversity, but different preferred diagnostic criteria

3 can lead to very different delineations. For instance, named primate species have more than

4 doubled since 1982. Such increases have been termed "taxonomic inflation" and have been

5 attributed to the widespread adoption of the 'phylogenetic species concept' (PSC) in preference to

6 the previously popular 'biological species concept' (BSC). Criticisms of the PSC have suggested

7 taxonomic inflation may be biased toward particular taxa and have unfavourable consequences for

8 conservation. Here, we explore predictors of taxonomic inflation across primate taxa since the

9 initial application of the PSC nearly 40 years ago. We do not find evidence that diversification

10 rate, the rate of lineage formation over evolutionary time, is linked to inflation, contrary to

11 expectations if the PSC identifies incipient species. We also do not find evidence of research effort

12 in fields where work has been suggested to motivate splitting being associated with increases in

13 species numbers among genera. To test the suggestion that splitting groups is likely to increase

14 their perceived risk of extinction, we test whether genera that have undergone more splitting have

15 also observed a greater increase in their proportion of threatened species since the introduction of

16 the PSC. We find no cohesive signal of inflation leading to higher threat probabilities across

17 primate genera. Overall, this analysis sends a positive message that threat statuses of primate

18 species are not being overwhelmingly affected by splitting in line with what has recently been

19 reported for birds. Regardless, we echo warnings that it is unwise for conservation to be reliant on

20 taxonomic stability. Species (however defined) are not independent from one another, thus,

21 monitoring and managing them as such may not meet the overarching goal of conserving

22 biodiversity. 
24 KEYWORDS: species; phylogenetic species concept; primates; taxonomic inflation; conservation

\section{INTRODUCTION:}

"Species" are an integral unit of biodiversity used across many sub-disciplines of biology, yet how scientists define species has been subject to change. New species definitions have led to dramatic shifts in the number of species listed in many taxonomic groups throughout time. Notably, in the last 40 years, the widespread popularization of the 'phylogenetic' or 'diagnostic' species concept (PSC) (Cracraft, 1983; Isaac, et al., 2004; Cotton, et al., 2016) has led to large increases in species numbers across many clades (Agapow, et al., 2004). through the late nineteenth to twentieth century. One notable phenomenon is the considerable decrease in diagnosed species that occurred following the rapid adoption of the polytypic species concept beginning in the 1890 's. The polytypic species concept emphasizes that species should be inclusive and that one should delineate taxa that resemble one another as subspecies (Groves, 2014). The popularization of the polytypic species concept was eventually accompanied by the widespread adoption of the 'biological species concept' (BSC) beginning in the early 1960's (Groves, 2014). The BSC defines species as populations/meta-populations that do not interbreed with other populations/meta-populations under natural conditions (Mayr, 1963; Groves, 2014).

44 While this definition has been subject to revisions, the central premise of the BSC is that reproductive barriers are key to diagnosing species (Groves, 2014). The BSC was widely accepted 
47 stability for vertebrates from the 1960's to 1980's (Isaac, et al., 2004). However, various criticisms

48 of the BSC did emerge, the most notable being the practical difficulty of diagnosing species under

49 the BSC because of the need for information on reproductive barriers. Closely related lineages

50 sharing parapatric (i.e., separate but contiguous) distributions are especially difficult to diagnose

51 under the BSC often due to limited records of potential mating interactions or of hybrid forms

52 (Tattersall, 2007). In these cases, genetic evidence is required to show that gene pools are linked,

53 e.g., actively reintegrating after being geographically separated (Tattersall, 2007). Collecting such

54 evidence in every case of parapatry would be a daunting task, and these critiques suggested the

55 need for a species concept with higher diagnostic power.

57 In the last 40 years the phylogenetic species concept (PSC) (Cracraft, 1983; Isaac, et al., 2004) has been widely popularized in vertebrate taxonomy due to its diagnosable advantages over the BSC. Under the PSC, a species is diagnosed as the smallest population or meta-population that is distinct in heritable differences from other populations or meta-populations (Cracraft, 1983; Groves \&

61 Grubb, 2011; Groves, 2014). According to its proponents, the PSC's emphasis on diagnosable 62 evidence gives it an advantage over other species concepts because it allows users to establish a scientific proposition that is testable. As a result, shortly after being proposed influential

64 taxonomists quickly advocated for use of the PSC over the BSC (see, e.g., Donoghue, 1985) and thus, the PSC began being used almost immediately after its inception.

67 Although the PSC offers diagnostic advantages over the BSC, it has also been subject to criticism, notably, for its tendency to split species into a range of less-inclusive units compared to previous 
70 PSC many populations previously recognized as subspecies or morphological variants have been

71 elevated to the full species status, resulting in a large increase in the number of listed species. For

72 instance, 181 species of primates were listed by Honacki et al. (1982), one year prior to Cracraft's

73 proposal of the PSC (Cracraft, 1983). Today the IUCN (International Union for Conservation of

74 Nature) lists over 500 distinct primate species (Estrada, et al., 2017). Some families (e.g.,

75 Cheirogaleidae and Indriidae) have more than tripled in species numbers since the introduction of

76 the PSC (see Figure 1). While some new species have been added as a result of new discoveries,

77 a majority are populations previously identified at a lower taxonomic level that have since been

78 elevated to species status following the application of the PSC (Tattersall, 2007). This trend of the

79 PSC increasing species numbers by raising taxonomic statuses has been referred to as 'taxonomic

80 inflation' (Isaac, et al., 2004; Rylands \& Mittermeier, 2014). Taxonomic inflation has been

81 criticized for being non-random, and biased toward certain taxonomic groups (Isaac, et al., 2004).

82 

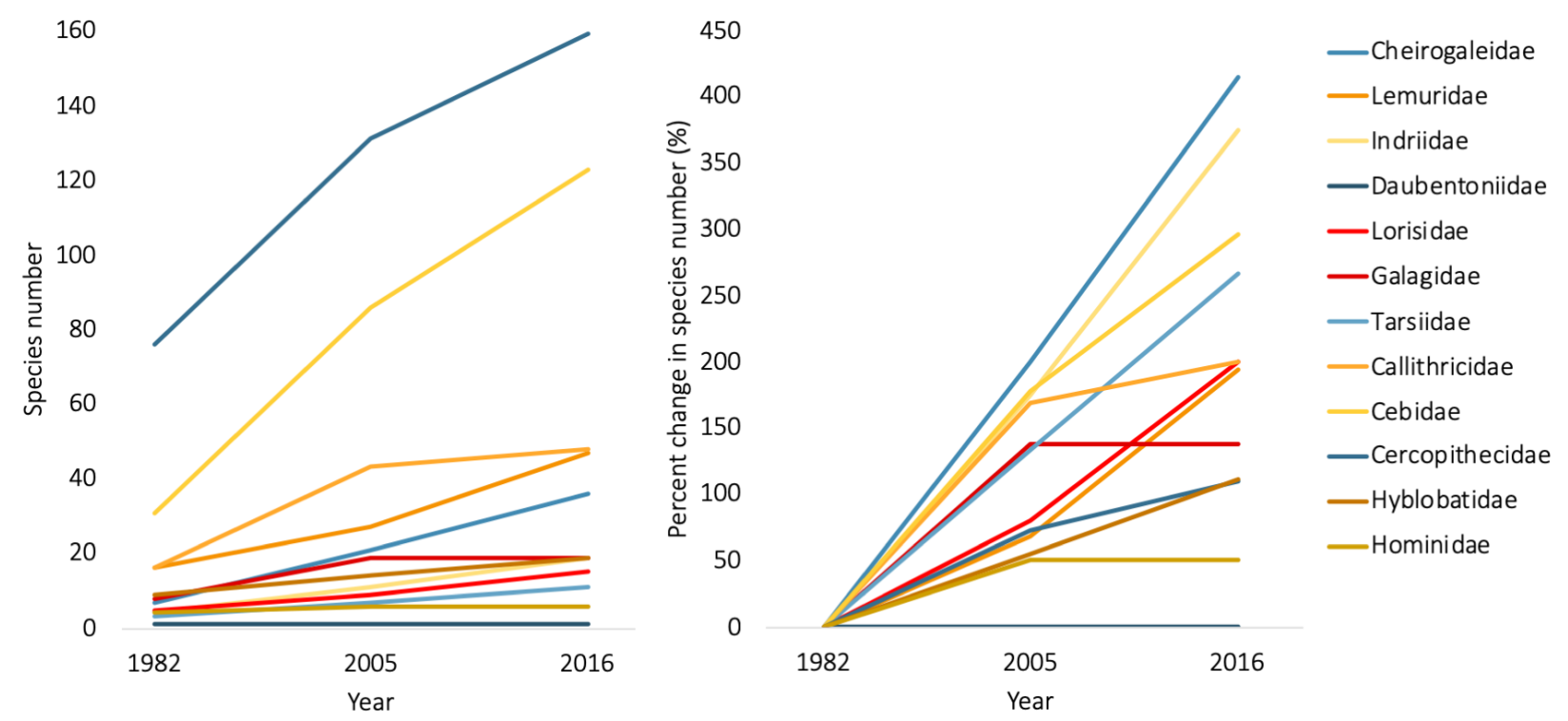

$87 \quad$ Figure 1: Species numbers and percentage change in species numbers for historic primate families recorded at three time points by Honacki et al. (1982), Wilson \& Reeder (2005) and the IUCN species list from 2016 (documented in Estrada, et al., 2017).

Disproportional splitting among taxonomic groups could have several causes. Variation in the number of new species described under the PSC across taxa could be driven by variation in the rate at which lineages evolve such that phylogenetic species are tracking cryptic diversity or incipient species formation among rapidly evolving taxa. In this case, new species listed under the PSC might point to situations where there is a discordance between patterns of genetic change and the evolution of gross morphological changes used by traditional taxonomists. This could be due to ecology - if some lineages are diversifying along ecological axes that we do not notice - or due to demographics - if some lineages have ecologies and/or histories that lead to faster local genetic coalescent times and so diagnosability. Under either of these scenarios, variation in inflation would simply reflect underlying biological reality. However, it is also possible that splitting is driven by other factors that may indeed be prone to bias. Critics have argued that much inflation is artificial, 
102 reflecting major shortcomings of applying the PSC and a reliance on insufficient data (Zachos, et 103 al., 2013; Zachos \& Lovari, 2013). Zachos et al. (2013) provides evidence suggesting unwarranted 104 splitting in select cases, advocating that the PSC has been taken to a "molecular extreme". In one 105 cited example (Zachos, et al., 2013), Sumatran tigers (previously Panthera tigris sumatrae, raised to Panthera sumatrae) were split by Cracraft et al. (1998) and then subsequently split into an even

107 greater number of species by Mazák \& Groves (2006), based on diagnosable mitochondrial and 108 craniometrics differences, respectively. By this logic, groups may continue to be split as an 109 increasing amount of molecular work leads to the continual identification of increasingly exclusive 110 diagnostic features such that we are likely to find more PSC species the more we look for them.

112 It is also possible that conservation interest in particular groups could motivate splitting. Limited 113 funding for conservation research is increasingly focused on 'biodiversity hotspots' and it has been 114 suggested that researchers could have a vested interest in declaring taxa in these regions as endemic 115 species (Karl \& Bowen, 1999; Isaac, et al., 2004). There is evidence from some charismatic groups 116 that taxa receive more conservation attention and funding when comprised of multiple, small, and 117 taxonomically distinct populations (e.g., African apes; Stanford, 2001; Oates, 2006; Gippoliti \& 118 Amori, 2007). Species lists are often used to determine which groups should receive conservation 119 attention (Mace, 2004) and changing the way we define species can also change which groups 120 receive action.

122 Along with being potentially biased toward certain taxonomic groups and related to the point made 123 above, splitting under the PSC has been suggested to result in individually more imperilled 124 populations which could negatively impact conservation agendas (Agapow, et al., 2004; Isaac, et 
125 al., 2004; Morrison, et al., 2009; Zachos, 2015; Robuchon, et al., 2019). One criterion used by the

126 IUCN to classify species as imperiled is population size: species may be designated "Vulnerable"

127 if there are fewer than 1000 mature individuals found in the wild and "Endangered" if there are

128 fewer than 250 (Agapow, et al., 2004; Frankham, et al., 2012). Thus, splitting one species into

129 several new species may result in one or more receiving a (more) imperilled status (Agapow, et

130 al., 2004; Isaac, et al., 2007). This could lead to seemingly rare but poorly-defined species being

131 prioritized over well-defined and perhaps biologically more distinctive species (Pillon \& Chase,

132 2007). Recent evidence suggests species splitting is not a driver of threat status for birds (Simkins,

133 et al., 2020), however, it is unknown whether these findings are generalizable across other

134 taxonomic groups (Garnett \& Thomson, 2020).

136 Here, we set out to better understand the causes and consequences of taxonomic inflation in

137 primates. We test (i) whether taxonomic inflation is associated with the amount of research being

138 done in fields suggested to motivate splitting, or with a lineage's underlying diversification rate,

139 and (ii) whether rates of inflation dictate which groups are considered to be most imperilled.

140 Because many newly described primate lineages have previously been described as subspecies or

141 subpopulations of multiple closely related BSC species, we ask these questions at the genus level.

143 To explore our first question regarding predictors of inflation, we consider whether biological

144 factors or measures of human-induced bias explain increases in species numbers. To test potential 145 human-induced biases in splitting, we consider estimates of research effort for each taxon, and 146 predict that more research done on a given taxon may be associated with more splitting. We 147 examine research effort in two fields: molecular genetics (since molecular work could cause 
148 species to be split continuously as finer molecular distinctions are made) and conservation (since

149 splitting has been suggested to be motivated by conservation interests). To explore possible

150 biological explanations for inflation trends, we test to see if inflation is explained by diversification

151 rate, using a diversification measure designed to be uncoupled from changes in species naming

152 conventions (see Methods). Lineages with high recent diversification rates are expected to contain

153 more incipient or cryptic species than lineages diversifying at a lower rate, such that diversification

154 rate might be expected to correlate positively with inflation.

156 To explore our second question linking inflation and risk, we test whether inflation is associated

157 with a change in the number of threatened species listed in genera through time. Ideally we would

158 look at changes in a weighted measure of threat score that differentiates between threat categories

159 of varying severity (e.g., using the Red List Index (RLI); Butchart, et al., 2007; Bubb, et al., 2009);

160 however, criteria for inclusion in Red List threat categories have changed considerably over time,

161 meaning weights assigned to categories for RLI calculations do not match up with categories used

162 when the PSC was first introduced (e.g., see statuses in IUCN Conservation Monitoring Centre,

163 1986). Because of these changes in Red List criteria, we instead ask whether clades with species

164 that have been split more frequently have observed a greater increase in their proportion of

165 threatened species (defined below) through time in comparison to those which have been split less

166 frequently. We predict that if splitting is driving an increase in threatened species, we should

167 observe a positive association between inflation and change in the proportion of threatened species

168 listed in primate genera. 


\section{Data}

172 To measure the number of primate species that were described before the introduction of the PSC,

173 we used the last pre-PSC taxonomy, that of Honacki et al. (1982). This taxonomy contains 181

174 species and is considered a reliable estimate of the number of species thought to exist during the

175 popularity of the BSC (see Rylands \& Mittermeier, 2014). We then recorded if each species in this

176 taxonomy was historically considered to be threatened by referencing the most complete IUCN

177 Red List published around the same time (IUCN Conservation Monitoring Centre, 1986). This

178 taxonomy was contrasted with the IUCN taxonomy from 2016 and attendant data documented in

179 Estrada et al. (2017), which lists 503 species. For each of these 503 species we noted their

180 taxonomic placement (genus and family), whether or not they were considered threatened

181 (VU=Vulnerable, EN=Endangered, or $\mathrm{CR}=$ Critically Endangered), and biogeographic region. For

182 each species described by the IUCN in 2016 that was not listed in Honacki et al. (1982), we scored

183 whether the species was a "de novo" species description (Burgin, et al., 2018), i.e., 40 cases where

184 a new species had not been previously formally identified as a subspecies or subpopulation of 185 another species prior to splitting.

187 We compiled all the species listed by the IUCN in 2016 into 12 families and 50 genera found in

188 Honacki et al. (1982). Family name "Callimiconidae" in Honacki et al. (1982) was not used as this

189 taxon has since been recognized as a genus of the larger family "Callitrichidae" (Wilson \& Reeder,

190 2005). Rungwecebus kipunji was removed from the IUCN species list from 2016 as it represents a

191 newly discovered genus that does not collapse into any of the genera provided by Honacki et al.

192 (1982). 
194 Research effort in the fields of molecular genetics and conservation were estimated for each genus

195 through a literature review of publications in the Web of Science Core Collection published

196 between 1983 (the year the PSC was first proposed) and 2016. To obtain papers in the field of

197 conservation we searched the genus name AND "conservation" (hereafter "conservation research

198 effort'). To obtain papers in the field of molecular genetics we searched for genus name AND the

199 following terms: "mitochondrial DNA" OR "barcoding" OR "bar-coding" OR "cytochrome b" OR

200 "phylogeography" OR "microsatellites" OR "micro-satellites" OR "population genetics" OR

201 (species AND genetics) OR (taxonomy AND genetics) OR "SNP" (hereafter "molecular genetics

202 research effort'). All hits from these searches were reviewed and irrelevant papers were eliminated

203 (see supplementary materials; Tables S1 and S2). In this study we used the genera listed in Honacki

204 et al. (1982) $(n=50)$, many of which have since been further separated into multiple genera. Thus,

205 when appropriate we included new genus names in addition to those listed by Honacki et al. (1982)

206 in the literature search (see Table S3). Additional details on how searches were conducted are

207 provided in the supplementary materials.

208

Diversification rate was estimated with the method-of-moments approach described in Magallon

211 using this method often rely on species numbers as their estimate of taxa richness, meaning that

212 diversification rate estimates are inherently biased by the inflation phenomenon we are studying

213 (i.e., highly inflated genera will receive disproportionally high diversification rates). Therefore,

214 richness scores for our diversification rate calculations were determined as the counts of the well-

215 resolved "lineages" described in Creighton et al. (2021). These lineages were determined by

216 creating a time cut-off in the 10kTrees consensus primate phylogeny (Arnold, et al., 2010) in an 
217 attempt to eliminate very young PSC species descriptions and obtain a consistent (unbiased)

218 estimate of diversity across clades. These lineages were assigned to each of the 50 genera described

219 in Honacki et al. (1982). Diversification rate was then estimated by taking the natural log lineage

220 richness for each genus and dividing by the stem age of that genus (Magallon \& Sanderson, 2001).

221 Stem ages for each genus were extracted from the 10kTrees consensus phylogeny (version 3)

222 (Arnold, et al., 2010) trimmed to contain a single branch representing each genus. During this

223 process, there were several instances where genera described in Honacki et al. (1982) were non-

224 monophyletic within the more recent primate phylogeny we used (Arnold, et al., 2010), making it

225 unclear how to assign a divergence date for these clades. We therefore removed eight genera from

226 the analyses where diversification rate was a variable of interest: Presbytis, Lemur, Galago,

227 Cebuella, Cercocebus, Cercopithecus, Papio, and Pygathrix. We discuss the limitations of having

228 to remove these genera from these analyses in the discussion.

230 Analysis

231 To test our questions about the predictors of species-splitting and its consequences for 232 conservation, we fit a series of linear effects and mixed effects models. We note that the response 233 variables used in these analyses (i.e., measures of inflation and extinction risk) are likely to be 234 phylogenetically clustered, and phylogenetic models could be used to account for this influence of 235 phylogeny; however, many genera listed by Honacki et al. (1982) are non-monophyletic, making 236 it unclear how to designate them a single branch in modern phylogenies (see discussion above on 237 diversification rate). Importantly, after accounting for regional differences, family contributed 238 little to no variance in any of our models, indicating that phylogenetic relationships at that level 
were not confounding our results. We provide further discussion on model choice in the supplementary materials.

Data were analysed using R version 4.0.5 (R Core Team, 2021).

\section{Predictors of Inflation}

245 To determine if measures of potential human bias (i.e., research effort) or diversification rate 246 explain discrepancies in inflation across taxa, we tested to see if these variables were significantly associated with the number of species added to primate genera since the introduction of the PSC while controlling for the original number of described species (i.e., species number prior to the

249 PSC) and region. We fit three generalized linear mixed effects models with Poisson distributions

250 using the lme4 package in R (Bates, et al., 2014), and obtained p-values using the lmerTest package

251 (Kuznetsova, et al., 2017). In these models the response variable was the number of species assigned to a given genus by the IUCN in 2016 that had not been previously described by Honacki et al. (1982). Each model had either conservation research effort, molecular genetics research

254 effort or diversification rate included as a fixed effect, as well as region and the number of species 255 in the genus per Honacki et al. (1982) to control for their effects on inflation. The natural logarithm 256 of the number of species listed for each genus in Honacki et al. (1982) was included as both a 257 linear and a quadratic term following inspection of raw plots and plots against scaled residuals 258 from the simulation output. A square-root transformation was used on molecular genetics research 259 effort to decrease the impact of outliers on model fit. To assist with model stability and 260 convergence, we scaled all continuous variables in the model to have a mean of zero and standard 261 deviation of one (Becker, et al., 1988). Mainland Africa and Asia (hereafter mainland Africa + 
Asia) were grouped together and served as the baseline region in our models based on previous studies that have shown that the taxonomy of primates from these regions has been relatively stable compared to Madagascar and the Neotropics (e.g., Isaac \& Purvis, 2004; Isaac, et al., 2004;

265 Tattersall, 2007). We also chose to group Asia and Africa together because one genus (Macaca) is found in both regions. Genus ID was included as a random effect in these models to correct for

267 overdispersion. Family (nested within region) was originally included as a random effect but 268 contributed very little to model fit and created issues with convergence due to overfitting, and thus 269 was dropped from the final models. We tested potential interaction terms with all variables and 270 region to test for regional effects, but none were significant and so these terms were also dropped

271 from the final models. Before running models with other predictors included, we also ran a model

272 including only the linear and quadratic terms for the number of species listed for each genus in 273 Honacki et al. (1982) as predictors to assess their association with inflation in the simplest model.

274 We ran all models a second time after removing the 40 de novo species from our response (Tables 275 S4, S5 and S6). We checked model assumptions and fit by plotting residuals versus the fitted 276 values and versus each covariate in the model. Residual plots and analyses with the Diagnostics 277 for Hierarchical Regression Models (DHARMa) R package (Hartig, 2017) indicated acceptable 278 model fits.

281 To test whether taxonomic inflation over time is associated with a change in the proportion of 282 threatened species within genera, we conducted a two-step (hierarchical) analysis on species' 283 threat probability between 1982 to 2016. We first fit a generalized linear mixed effects model with 284 a binomial distribution for the number of threatened and non-threatened species within a genus 
using the lme4 package in R (Bates, et al., 2014). In this first model, the response estimated the proportional counts of species at risk within genera (equivalent to the per-species probability of threat) in the periods of 1982 and 2016. Predictors for this model included fixed effects for the time period (the baseline of 1982 and the change to 2016), the region that encompasses each genus' natural distribution (Madagascar, Neotropics, mainland Africa + Asia), and an interaction between region and time period to account for geographic differences in threat probabilities through time.

291 Genus identity was included as a random intercept, to account for repeated measures in 1982 and 292 2016, and as a random slope with time period to account for differences in changes to threat 293 probabilities among genera. As discussed above, we included the taxonomic rank of family (nested 294 within region) as a random effect but found that the variance in threat probabilities among families 295 was minimal and that including this term also generated model convergent issues; given this, we subsequently omitted family from our models. Residual plots and analyses with the DHARMa R 297 package (Hartig, 2017) indicated acceptable model fits for the final model. From this first model 298 describing genus level changes in threat probabilities, we then used the REextract function 299 implemented in the merTools package in R (Knowles \& Frederick, 2020) to extract the genus level 300 random slopes for time period and their associated standard errors, giving us an estimate of the 301 varying effect of change in threat probability among genera (while conditioning on regional trends) 302 between 1982 and 2016. We then fit a linear model where varying effect of change in threat 303 probability from 1982 to 2016 was the response, and the proportional change in species within 304 each genus (i.e., the number of new species in the IUCN species list from 2016 / original number of species in Honacki et al. (1982) - our measure of "inflation") - was the predictor. We weighted each estimate of genus level change in threat probability by its standard error $(\mathrm{w}=1 / \mathrm{SE})$ to 307 propagate the error of model estimated random effects. We ran this model a second time after 
removing de novo species descriptions from our response (Table S7). Raw data used for these tests are visualized in Figure 2.

311 Plots of the raw data indicated that patterns in inflation, the proportion of species at risk in primate

312 genera today, and the changes the proportion of threatened species in primate genera between 1982

313 and 2016 show regional differences (Figures S1 and S2). We ran a subsequent set of models to test

314 for regional variation in the effect of taxonomic inflation. In this analysis, we removed region from

315 the first generalized linear mixed effects model and subsequently included region interacting with taxonomic inflation as a predictor of the varying effect of change in genus threat probability in the second-order model. However, interaction effects were not significant in this model and thus we only considered the results of the first set of models reported above.

\section{RESULTS:}

\section{Predictors of Inflation}

322 None of our measures - conservation research effort $(\beta=-0.220 ; p=0.180$; Table S4), molecular 323 genetics research effort $(\beta=-0.188 ; \mathrm{p}=0.212$; Table S5) nor diversification rate $(\beta=0.132 ; \mathrm{p}=$

324 0.477; Table S6) - were significantly associated with taxonomic inflation of primate genera.

325 Removing de novo species did not impact this pattern of results (Tables S4, S5 and S6). Notably, 326 in addition to sharing a significant linear relationship with inflation as expected (Tables S4, S5 and

327 S6), the quadratic term added for the original number of species in 1982 was significant in the 328 model with diversification rate, indicating a downwardly concave association with inflation $(\beta=$ 1.890; $p=0.042$; Table S6); this term approached statistical significance in models that included 330 conservation or molecular genetics research effort as predictors $(\beta=-1.529 ; p=0.072$; Table S4 
331 and $\beta=-1.626 ; p=0.057$; Table S5), indicating that the positive increase in splitting by initial genus

332 size decelerates as genera become very large, even after accounting for other factors. We note that

333 this quadratic term was also significant without other predictors (i.e., research effort or

334 diversification rate) present in the model, both with de novo species included in the response ( $\beta=$

$335-2.268 ; \mathrm{p}=0.011)$ and without de novo species included in the response $(\beta=-2.086 ; \mathrm{p}=0.013)$.

337 Inflation and Threat Score

338 Looking at the raw data on inflation and proportion of species at risk for all genera, there was no

339 clear indication that highly inflated genera have observed a disproportional increase in perceived

340 extinction risk (Figure 2). 
a)

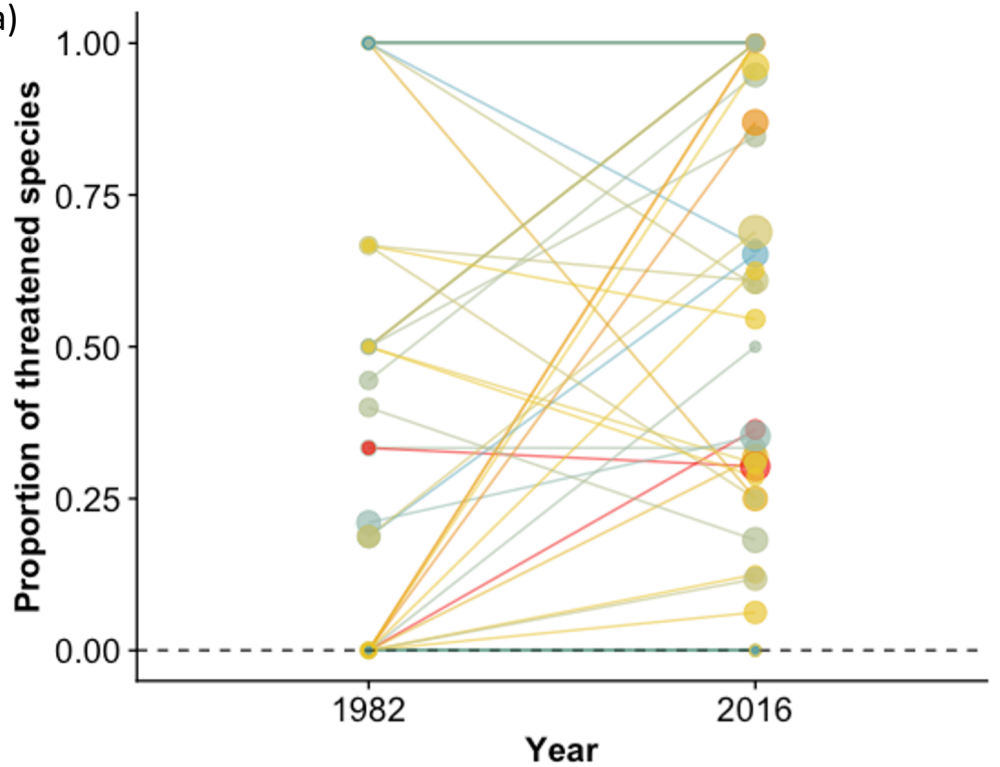

b)

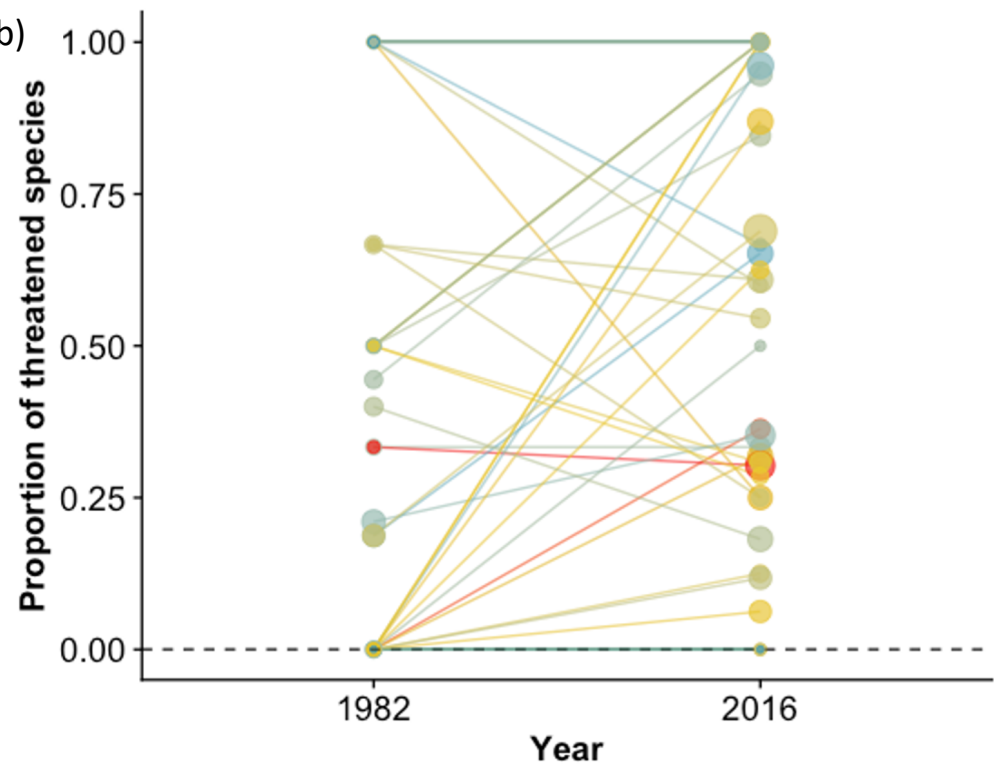

Number of species

- 10

- 20

30

40

Sqrt of inflation (with de novo species)

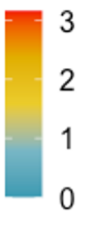

Number of species

- 10

- 20

30

40

Sqrt of inflation (without de novo species

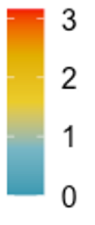

$343 \quad$ Figure 2: Scatterplots with trendlines showing the change in the proportion of species identified

344 as being threatened in primate genera in 1982 and 2016 painted by inflation a) including de novo

345 species, and b) excluding de novo species. Total number of species in each genus is indicated by 346 point size. 

errors on varying effect of change in threat probability values. pattern of results (Table S7; Figure S3).

Results from our GLMM confirmed that inflation did not predict a change in threat score through time $(\beta=0.002 ; p=0.975$; Table S7; Figure 3$)$. Removing de novo species did not impact this

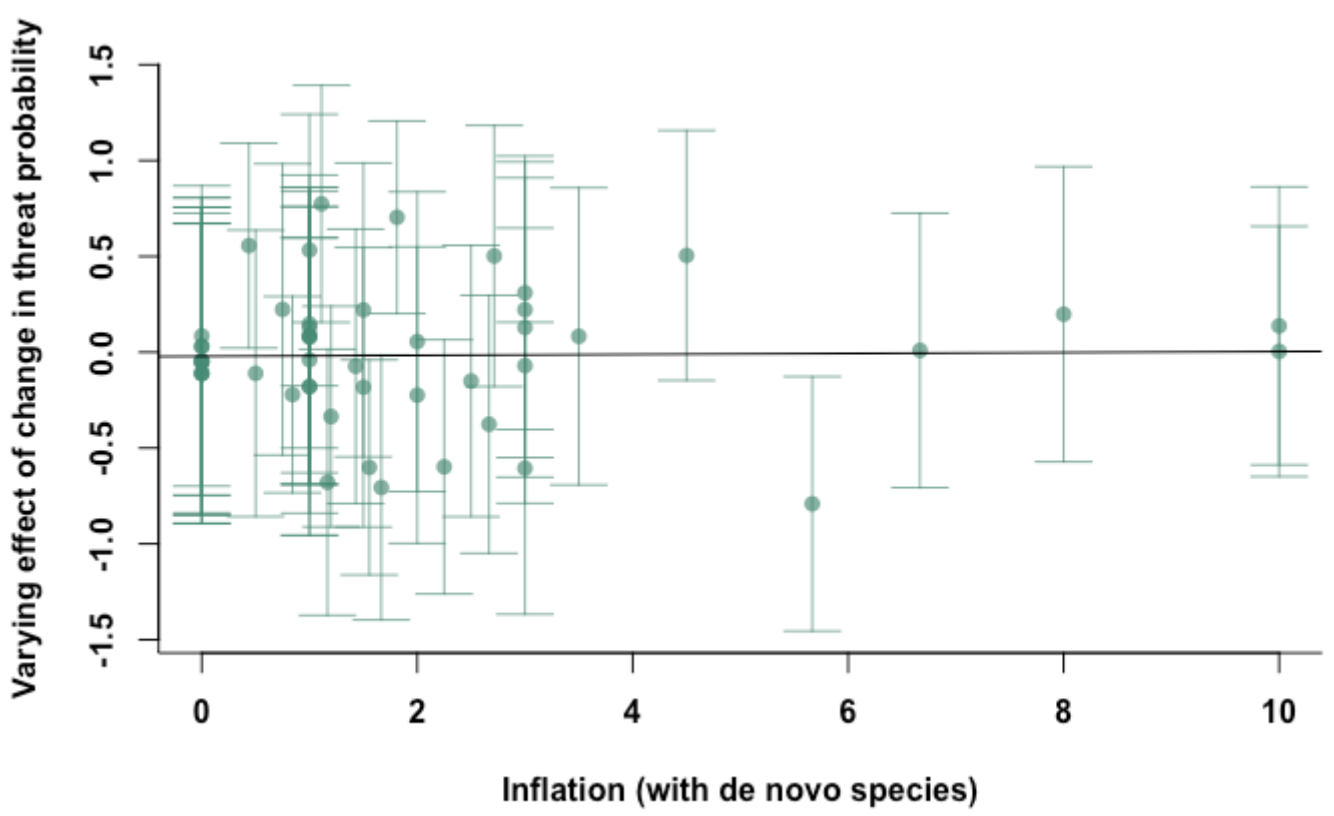

Figure 3: Inflation versus varying effect of change in threat probability (i.e., our measure of genus level change in threat probability) $(\beta=0.001 ; p=0.975$; Table $S 7)$. Vertical bars indicate standard DISCUSSION:

358 Our results support neither a strong biological nor a strong interest-driven mechanism for variation 359 in new species designations across primate genera. If the naming of new PSC species captures 360 incipient speciation, we predicted that diversification rate might predict inflation. However, we 361 found no evidence for this as diversification rate was not a significant predictor of inflation in our 362 models. We also tested the prediction that splitting is motivated by conservation interest (Karl \& 
363 Bowen, 1999; Isaac, et al., 2004) or by increasing molecular research within certain taxa, with for example, more new species designations arising as more population genetics work is done (Zachos, et al., 2013; Zachos \& Lovari, 2013). However, we found no evidence that research effort in either

366 of these areas was associated with inflation across genera. We did find that the positive increase

367 in splitting by initial genus size decelerates as genera approach the largest sizes. This could indicate

368 that inflation is being driven by something not captured by our models. Therefore, future studies

369 could aim to elucidate the origins of this decelerating association.

371 While inspection of the raw data suggested that genus level measures of inflation, threat

372 probability, and change in threat probability all varied by region, we found that there was no

373 cohesive signal of inflation leading to higher threat probability across these genera and regions.

374 Therefore, while there are idiosyncratic regional differences in both primate inflation and threat

375 probability there is no evidence for causal link between the two. While in some cases splitting may

376 lead populations to be considered more imperilled, overall, this analysis sends a positive message

377 that primates species threat statuses are not being overwhelmingly affected by splitting in line with

378 what has been reported for birds (Simkins, et al., 2020).

380 It should be considered that our analyses do have some limitations. First, quantifying research

381 effort of any kind is difficult due to the abundance of work published in different media. As such,

382 our estimates of research effort in conservation and molecular genetics may overlook some types

383 of research. More work should be done to determine if additional estimates of research bias can

384 explain increases in species numbers. For instance, cumulative funding estimates from various

385 sources (e.g., the IUCN, non-governmental organizations and regional governments) per taxon 
could be used as an additional or alternative measure of conservation interest. Second, it was necessary to remove eight non-monophyletic genera (some that have undergone substantial splitting) from those analyses that included diversification rate, leading to a considerable reduction in statistical power for those analyses. Third, due to changes in Red List criteria, species status, and lack of information about which species had been assessed in the 1980 s, we were not able to consider differences in threat status severity (e.g., Vulnerable versus Endangered) when considering associations between inflation and changes in threat score through time. This leaves room for the possibility that change in risk could be under- or over-estimated for certain genera. Future studies could look at the association between inflation and changes in weighted measures of threat score (e.g., RLI) over time using a more recent starting point (i.e., after 1982) where Red List criteria become stable and there is available information on which non-threatened species have been evaluated.

Overall, we do not find support for biological processes (i.e., diversification rate) or human imposed bias (i.e., measures of research effort) driving inflation across primate genera. We also find no cohesive signal of inflation leading to higher threat probabilities across primate genera. Generally speaking, relying on species as the central unit of conservation and primary object of biological study behooves taxonomy to remain stable, while changing ideas about the concept of species makes taxonomy inherently unstable (Mayr, 1996). We suggest that areas of research requiring consistent estimates of diversity (i.e., conservation) may benefit from either (i) adopting stable species criteria, i.e., that do not rely on increasingly fewer diagnostic distinctions; or, for applied conservation specifically (ii) shifting more resources toward regional management efforts that are less likely to be influenced by changing species designations. It is well-known that closely 
409 related species are more similar to one another than they are to more distantly related taxa. Thus,

410 treating all species independently and of equal weights in conservation listing may not lead to

411 desired outcomes (Redding \& Mooers, 2010). As of 2016, approximately 60 percent of all primate

412 species were threatened with extinction according to the IUCN Red List of Threatened Species

413 (Estrada, et al., 2017), making it imperative that conservation efforts are spent wisely to ensure

414 optimal conservation of primate biodiversity writ large.

ACKNOWLEDGEMENTS: This work was supported by the Natural Sciences and Engineering

417 Research Council of Canada (NSERC) Discovery Grants Program (SMR and AOM, grants \#2017-

41804720 and \#2019-04950) and the Canada Foundation for Innovation (CFI) (SMR, grant \#29433).

419 MJAC was supported by awards from the Biodiversity, Ecosystem Services, and Sustainability

420 (BESS) program and McGill University. We thank the Reader and Guigueno labs at McGill, Brian

421 Leung, Hans Larsson, William Wcislo, Isabella Capellini, and the Crawford Lab for Evolutionary

422 Studies at SFU for feedback on portions of the manuscript and study design. Special thanks to

423 Aiman Hadif for his assistance in collecting and organizing data and to Paul Sims and Dan

424 Greenberg for crucial statistical advice.

DECLARATIONS OF INTEREST: None.

428 Data and code accompanying the manuscript will be made available on publication through 429 Zenodo. 


\section{REFERENCES:}

Agapow, P. M., Bininda-Emonds, O. R., Crandall, K. A., Gittleman, J. L., Mace, G. M., Marshall, J. C., \& Purvis, A. (2004). The impact of species concept on biodiversity studies. The Quarterly Review of Biology, 79: 161-179.

Arnold, C., Matthews, L. J., \& Nunn, C. L. (2010). The 10kTrees website: a new online resource for primate phylogeny. Evolutionary Anthropology, 19: 114-118.

Bates, D., Maechler, M., Bolker, B., \& Walker, S. (2014). lme4: Linear mixed-effects models using Eigen and S4. $\mathrm{R}$ package version 1.1.21. https://cran.rproject.org/web/packages/lme4.

Becker, R. A., Chambers, J. M., \& Wilks, A. R. (1988). The new S language: A programming environment for data analysis and graphics. Wadsworth \& Brooks/Cole, Pacific Grove.

Bubb, P. J., Butchart, S. H. M., Collen, B., Dublin, H., Kapos, V., Pollock, C., Stuart, S. N., \& Vié, J. -C. (2009). IUCN Red List Index_-guidance for national and regional use. IUCN, Gland.

Burgin, C. J., Colella, J. P., Kahn, P. L., \& Upham, N. S. (2018). How many species of mammals are there? Journal of Mammalogy, 99: 1-14.

Butchart, S. H., Akçakaya, H. R., Chanson, J., Baillie, J. E., Collen, B., Quader, S., ... \& HiltonTaylor, C. (2007). Improvements to the Red List Index. PLoS One, 2: e140.

Cotton, A., Clark, F., Boubli, J. P., \& Schwitzer, C. (2016). IUCN Red List of threatened primate species. In S. A. Wich \& A . J. Marshall (Eds.), An introduction to primate conservation (p. 31-38). Oxford University Press, Oxford. 
Cracraft, J. (1983). Species concepts and speciation analysis. In R. F. Johnson (Ed.), Current ornithology (p. 159-187). Plenum Press, New York.

Cracraft, J., Feinstein, J., Vaughn, J., \& Helm-Bychowski, K. (1998). Sorting out tigers (Panthera tigris): mitochondrial sequences, nuclear inserts, systematics, and conservation genetics. Animal Conservation, 1: 139-150.

Creighton, M. J. A., Greenberg, D. A., Reader, S. M., \& Mooers, A. Ø. (2021). The role of behavioural flexibility in primate diversification. Animal Behaviour. Advance online publication.

Donoghue, M. J. (1985). A critique of the biological species concept and recommendations for a phylogenetic alternative. Bryologist, 88: 172-181.

Estrada, A., Garber, P. A., Rylands, A. B., Roos, C., Fernandez-Duque, E., Di Fiore, A., ... \& Rovero, F. (2017). Impending extinction crisis of the world's primates: Why primates matter. Science Advances, 3: e1600946.

Frankham, R., Ballou, J. D., Dudash, M. R., Eldridge, M. D., Fenster, C. B., Lacy, R. C., ... \& Ryder, O. A. (2012). Implications of different species concepts for conserving biodiversity. Biological Conservation, 153: 25-31.

Garnett, S. T., \& Thomson, S. A. (2020). Are the implications for conservation of a major taxonomic revision of the world's birds' simply serendipity? Animal Conservation, 23: 355-356.

Gippoliti, S., \& Amori, G. (2007). The problem of subspecies and biased taxonomy in conservation lists: the case of mammals. Folia Zoologica, 56: 113-117. 
474 Groves, C. P. (2014). Primate taxonomy: inflation or real? Annual Review of Anthropology, 43: $27-36$.

Groves, C., \& Grubb, P. (2011). Ungulate taxonomy. JHU Press, Baltimore.

Hartig, F. (2017). DHARMa: residual diagnostics for hierarchical (multi-level/mixed) regression models. R package version 0.3.0. https://cran.r-project.org/web/packages/DHARMa.

Honacki, J. H., Kinman, K. E., \& Koeppl, J. W. (1982). Mammal species of the world; a taxonomic and geographic reference. Allen Press, Inc. and Association of Systematic Collections, Lawrence.

Isaac, N. J., Mallet, J., \& Mace, G. M. (2004). Taxonomic inflation: its influence on macroecology and conservation. Trends in Ecology \& Evolution, 19: 464-469.

Isaac, N. J., \& Purvis, A. (2004). The 'species problem' and testing macroevolutionary hypotheses. Diversity and Distributions, 10: 275-281.

Isaac, N. J., Turvey, S. T., Collen, B., Waterman, C., \& Baillie, J. E. (2007). Mammals on the EDGE: conservation priorities based on threat and phylogeny. PLoS One, 3: e296.

IUCN Conservation Monitoring Centre. (1986). 1986 IUCN Red List of threatened animals. IUCN, Gland and Cambridge.

Karl, S. A., \& Bowen, B. W. (1999). Evolutionary significant units versus geopolitical taxonomy: molecular systematics of an endangered sea turtle (genus Chelonia). Conservation Biology, 13: 990-999.

Knowles, J. E., and Frederick, C. (2020). merTools: Tools for Analyzing Mixed Effect Regression Models. R package version 0.5.2. https://CRAN.R-project.org/package=merTools. 
Kuznetsova, A., Brockhoff, B., \& Christensen, H. B. (2017). lmerTest Package: Tests in Linear Mixed Effects Models. Journal of Statistical Software, 82: 1-26.

Mace, G. M. (2004). The role of taxonomy in species conservation. Philosophical Transactions of the Royal Society B: Biological Sciences, 359: 711-719.

Magallon, S., \& Sanderson, M. J. (2001). Absolute diversification rates in angiosperm clades. Evolution, 55: 1762-1780.

Mayr, E. (1963). Animal species and evolution. Belknap Press, Cambridge.

Mayr, E. (1996). What is a species, and what is not? Philosophy of Science, 63: 262-277.

Mazák, J. H., \& Groves, C. P. (2006). A taxonomic revision of the tigers (Panthera tigris) of Southeast Asia. Mammalian Biology, 71: 268-287.

Morrison, W. R., Lohr, J. L., Duchen, P., Wilches, R., Trujillo, D., Mair, M., \& Renner, S. S. (2009). The impact of taxonomic change on conservation: Does it kill, can it save, or is it just irrelevant? Biological Conservation, 142: 3201-3206.

Oates, J. F. (2006). Is the chimpanzee, Pan troglodytes, an endangered species? It depends on what “endangered" means. Primates, 47: 102-112.

Pillon, Y., \& Chase, M. W. (2007). Taxonomic exaggeration and its effects on orchid conservation. Conservation Biology, 21: 263-265.

R Core Team. (2021). R: A Language and environment for statistical computing. R Foundation for Statistical Computing, Vienna, Austria. http://www.R-project.org/.

Redding, D. W., \& Mooers, A. Ø. (2010). Can systematists help decide the relative worth of bits of biodiversity. The Systematist, 32: 4-8. 
Robuchon, M., Faith, D. P., Julliard, R., Leroy, B., Pellens, R., Robert, A., ... \& Pavoine, S. (2019). Species splitting increases estimates of evolutionary history at risk. Biological Conservation, 235: 27-35.

Rylands, A. B., \& Mittermeier, R. A. (2014). Primate taxonomy: species and conservation. Evolutionary Anthropology, 23: 8-10.

Simkins, A. T., Buchanan, G. M., Davies, R. G., \& Donald, P. F. (2020). The implications for conservation of a major taxonomic revision of the world's birds. Animal Conservation, 23: $345-352$.

Stanford, C. B. (2001). The subspecies concept in primatology: the case of mountain gorillas. Primates, 42: 309-318.

Tattersall, I. (2007). Madagascar's lemurs: cryptic diversity or taxonomic inflation? Evolutionary Anthropology, 16: 12-23.

Wilson, D. E., \& Reeder, D. M. (2005). Mammal species of the world: a taxonomic and geographic reference (vol. 1). JHU Press, Baltimore.

Zachos, F. E. (2015). Taxonomic inflation, the phylogenetic species concept and lineages in the tree of life-a cautionary comment on species splitting. Journal of Zoological Systematics and Evolutionary Research, 53: 180-184.

Zachos, F. E., Apollonio, M., Bärmann, E. V., Festa-Bianchet, M., Göhlich, U., Habel, J. C., ... \& Pertoldi, C. (2013). Species inflation and taxonomic artefacts-A critical comment on recent trends in mammalian classification. Mammalian Biology, 78: 1-6. 
bioRxiv preprint doi: https://doi.org/10.1101/2021.09.10.459781: this version posted September 11. 2021. The copvriaht holder for this preprint (which was not certified by peer review) is the author/funder, who has granted bioRxiv a license to display the preprint in perpetuity. It is made available under aCC-BY-NC-ND 4.0 International license.

536 Zachos, F. E., \& Lovari, S. (2013). Taxonomic inflation and the poverty of the Phylogenetic

537 Species Concept-a reply to Gippoliti and Groves. Hystrix, 24: 142-144. 